\title{
fMRI Compatible Haptic Interface Actuated with Traveling Wave Ultrasonic Motor
}

\author{
Markus Flueckiger, Matteo Bullo, *Dominique Chapuis, *Roger Gassert, Yves Perriard \\ Integrated Actuators Laboratory - LAI \\ *Laboratory of Robotic Systems - LSRO \\ Ecole Polytechnique Fédérale de Lausanne - EPFL \\ CH - 1015 LAUSANNE - SWITZERLAND \\ Phone : +41216936693 \\ Fax : +41216932050 \\ Email : markus.flueckiger@epfl.ch
}

\begin{abstract}
We are developing haptic interfaces compatible with functional Magnetic Resonance Imaging (fMRI) for neuroscience studies. The presented prototype with one rotary degree of freedom is actuated by a traveling wave ultrasonic motor operating under admittance control. Torque is sensed from the deflection of an elastic polymer probe via light intensity measurement over optical fibers. This concept allows us to place all electronic components outside the shielded MR room. Hence, the device can be used in conjunction with fMRI, providing torque and motion feedback simultaneously with imaging. Its compactness and simplicity facilitate the construction of multiple degree of freedom systems.
\end{abstract}

\section{INTRODUCTION}

\section{A. Interest of fMRI Compatibility}

Magnetic Resonance Imaging (MRI) is a standard technology in diagnostics [1] and advanced brain research [2]. Robots compatible with MRI [3], [4] and functional MRI (fMRI) [5] are required to develop assistive devices for intraoperative MRI and haptic interfaces for neuroscience studies. Investigating the adaptation of human motor control to virtual dynamic environments produced by such interfaces has brought major advances in neuroscience [6]. A robotic interface in conjunction with an MR scanner would enable neuroscientists to investigate the brain mechanisms involved in performing tasks with arbitrary dynamics [7], and therefore has the potential to become a critical tool in neuroscience and rehabilitation [8].

\section{B. fMRI Compatibility and Safety}

In addition to the standard requirements of medical robots, such as safety and sterilization issues, an fMRI compatible device must not disturb the scanning procedure. The robot motion should not have any adverse effect on the image, and in turn, the robot must not be affected by the scanner's magnetic field and RF pulses. A descriptive definition of MR compatibility can be found in [9], a resume of techniques for MR compatible robots is given in [10]. However, we must consider that functional imaging sequences are even more sensitive to perturbations of the magnetic field than standard diagnostic sequences. The MR safety of the robot requires that the machine should not unintentionally move from any magnetic attraction and no electromagnetic side effects should occur.

\section{Requirements for Neuroscience Studies}

To study neuromuscular response during dynamical interaction with humans performing movements, the haptic interface must deliver forces smoothly and fast enough to allow the rendering of any desired force field.

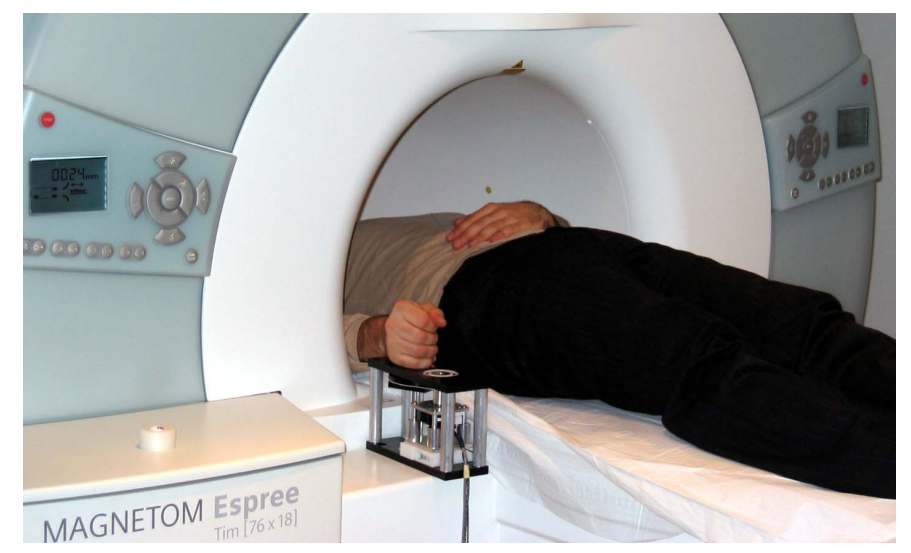

Fig. 1. Testing of the haptic interface prototype inside an MR scanner at the University Hospital in Basel.

The test procedures can be classified into two main types :

- Passive movement (from subjects point of view) : The haptic interface moves along a predefined trajectory, the subject, holding the output handle, follows this movement with his hand.

- Active movement (from subjects view) : The subject actuates the haptic interface and feels the torque feedback of a virtual environment.

\section{Prototype Design Issues}

\section{A. Mechanical Structure}

The main challenge we face in designing the mechanics of an MR compatible haptic interface are the strong static magnetic field and the fast switching magnetic field gradients which are present in an MR environment. As the structure will 
be exposed to a static magnetic field of typically 1.5 to 3 Tesla, only non-ferromagnetic materials can be used. Moreover, the amount of electrically conducting parts should be reduced to a minimum to avoid possible disturbance due to eddy currents, induced by the switching magnetic field gradients. Therefore, the polymer Polyoxymethylene (POM) was mainly used. Parts exposed to high stress are manufactured from aluminum.

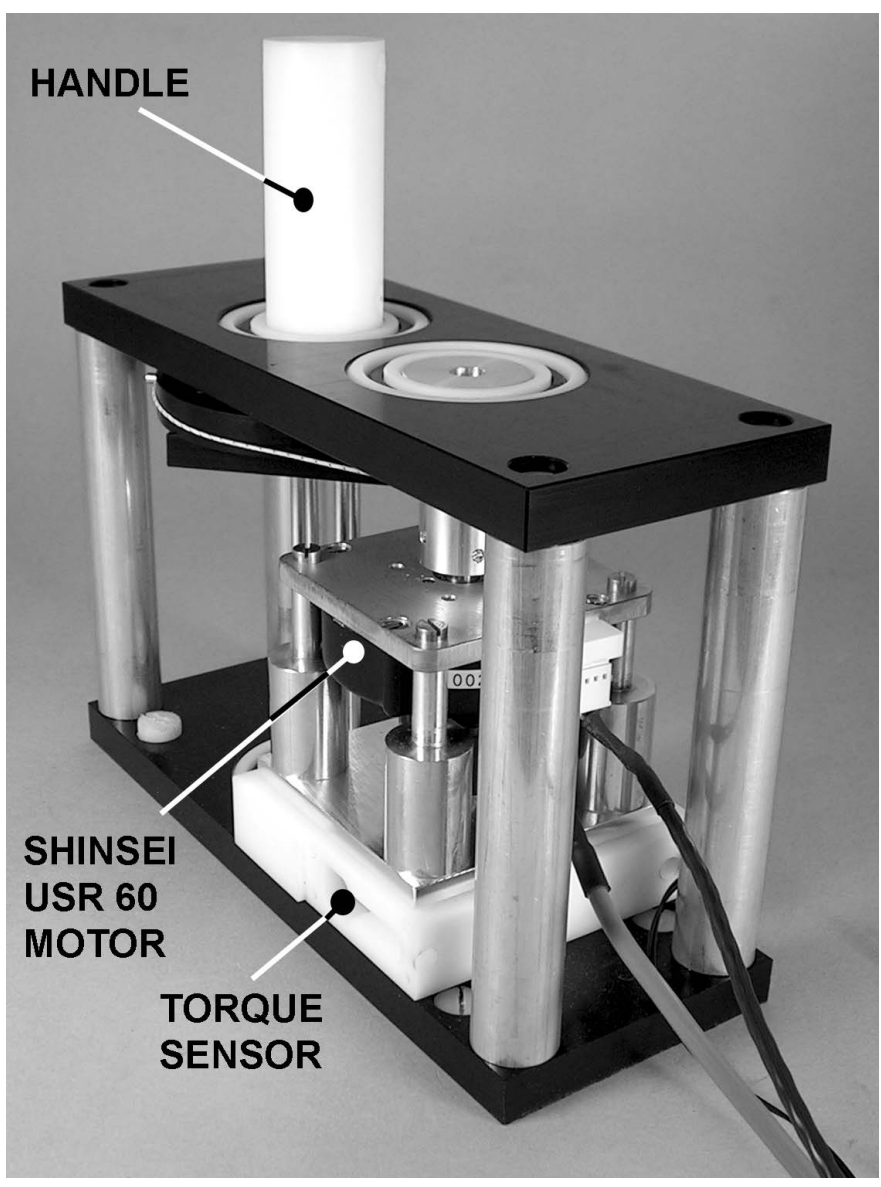

Fig. 2. Prototype of the MR compatible 1 DOF haptic interface.

\section{B. Motor Selection}

Supposing that the haptic interface must be a compact mechanical system whose actuator operates at the place of interest, which is at the entry of the scanner bore (i.e. where the hand of the subject is located), conventional electromagnetic motors cannot be used. We must choose an actuator that, on the one hand, is able to operate within an MR environment, on the other hand, does not disturb the imaging in any way. The MR compatible version of the Shinsei URS60 traveling-wave ultrasonic motor (TUSM) was selected because it presents weak electromagnetic disturbances and is insensitive to high magnetic fields. Moreover, this actuator is very well adapted to the haptic interface application because it reaches it's maximum efficiency at high torque and low speed, the common range of operation in the present application. Further, it has a high power density compared to the conventional electromagnetic motors. Its holding torque (at rest, without feeding), however, will be a challenge for controller implementation. Estimation of output torque will not be practicable, which is why a torque sensor is needed. TUSM have been used for positioning tasks in surgical assist robots [11], [4] whose compatibility with intraoperative MRI has been tested successfully [9].

\section{Torque Sensing}

To allow accurate force measurement over a relatively long distance without any electronic circuitry, we use optical sensors with light transmitted through optical fibers to detect deflection of a polymer probe by reflected light intensity measurement. This torque sensor, described in [12] was adapted to the present application and is shown in figure 3 .

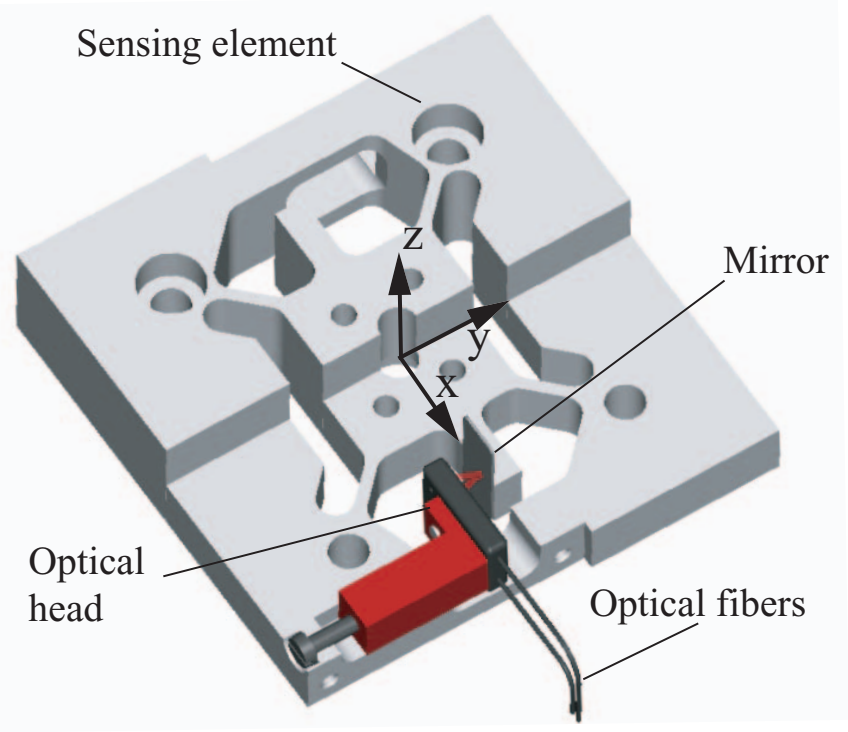

Fig. 3. Schema of the MR compatible torque sensor (with partial cut)[12].

\section{Position and Speed Sensing}

To determine the motor position we use an optical encoder ${ }^{1}$, which was delivered with the USR60 motor. Its 500 increments per turn, using both channels, result in a resolution of 2000 pulses per turn. In addition we are able to deduce speed information by using the internal timer of the DSP. An adequate conversion factor finally allows us to calculate the motor speed in rpm.

\section{MOTOR CONTROL}

TUSM are friction drives. Hence, they are nonbackdriveable. Consequently, there is no control parameter to directly impose torque at the output. We therefore use an admittance control paradigm (section III-A) where motion is controlled to achieve force feedback control.

\section{A. Admittance Control Strategy}

In the present case the goal of the admittance control is to render the desired force field of a virtual target environment

\footnotetext{
${ }^{1}$ HEDS-5540, HP
} 


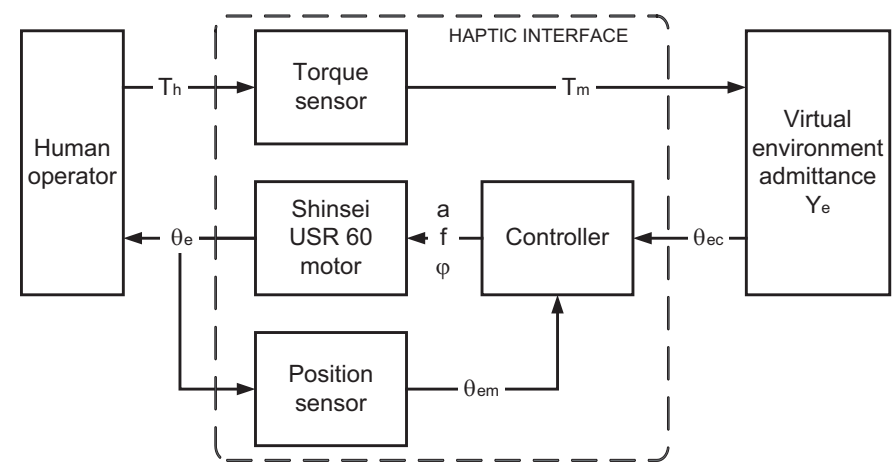

Fig. 4. In admittance control, the controller receives the torque that the user exerts on the haptic interface $\left(T_{h}\right)$ as input, and reacts with motion $\left(\theta_{e}\right)$, which is computed by a discrete virtual environment model represented by its admittance $\left(Y_{e}\right)$.

at the output of the haptic interface as shown in figure 4 . We note the torque exerted by the human to the output handle of the haptic interface as $T_{h}$ and the position of the actuator as $\theta$. The corresponding law which relates applied torque and actuator position is specified by the admittance of the virtual environment $\left(Y_{e}\right)$ (equation 1)

$$
\theta_{e}=Y_{e} T_{h}
$$

The detailed control scheme is presented in figure 5. The external control loop generates the position command as a function of the applied torque and the admittance of the virtual environment (section III-D). The internal control loop consists of a position controller cascaded with a proportional integral speed controller that generates the control signal for the TUSM.

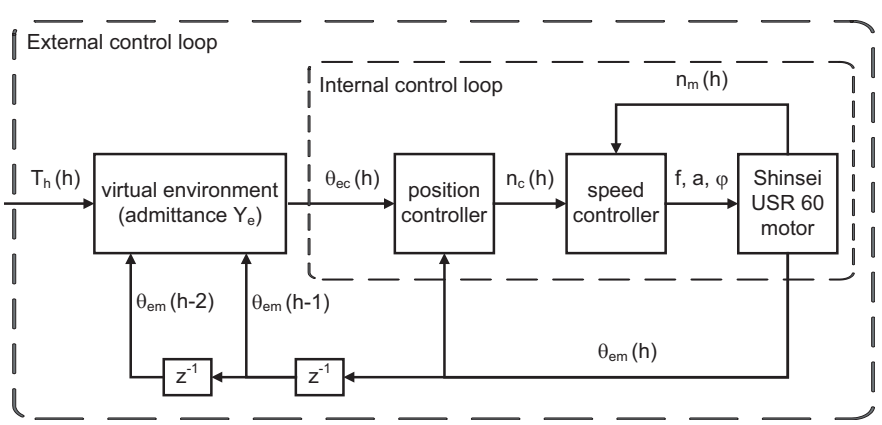

Fig. 5. Control diagram of the implemented admittance controller with position and speed feedback. The external admittance control loop generates the commanded position $\left(\theta_{e c}\right)$ as a function of applied torque $\left(T_{h}\right)$ and measured motor position $\left(\theta_{e m}\right)$ at time $(h-1)$ and $(h-2)$. In the internal control loop, the motor command values frequency $f$, amplitude $a$ and phase shift $\phi$ of the two supply voltages are generated.

\section{B. Speed Control}

Basically, the speed of the TUSM can be controlled by varying the frequency, the voltage amplitude and the phase difference of the two sinusoidal input waveforms. However, the TUSM suffers from severe system nonlinearities and parameter variations. Especially the drift of the resonance frequency due to motor heating presents a challenge. Therefore, it is not possible to use a basic PID type controller without any precaution. Adaptive control theory is a promising approach and research was done on polynomial [13], fuzzy logic [14] and neural network [15], [16] based adaptive controllers.

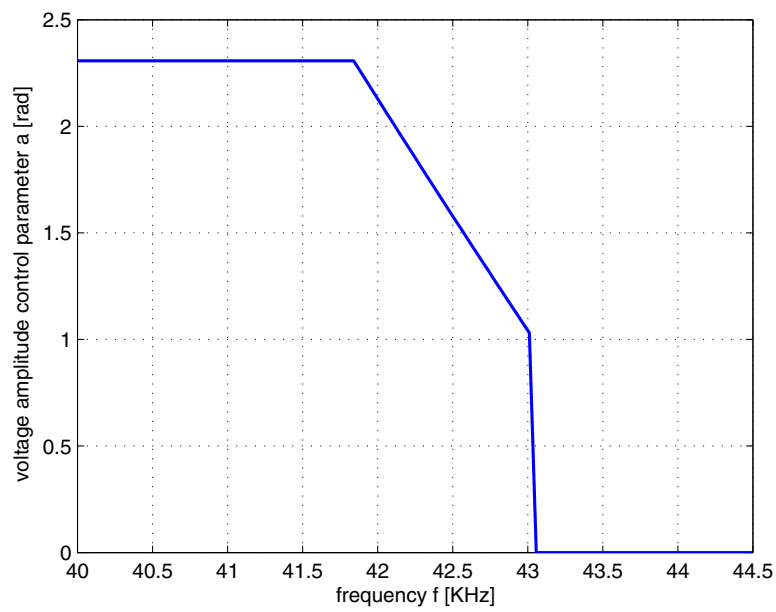

Fig. 6. The amplitude of the traveling wave is a function of frequency as shown in the graph.

We suggest a control approach by amplitude modulation of the supply voltage. We use the degree of freedom offered by the amplitude to linearize the frequency-speed characteristic. The optimal variation of the supply voltage making it possible to linearize the characteristic is obtained when the parameter of control $a$ is a function of the frequency according to the graph shown in figure 6 .

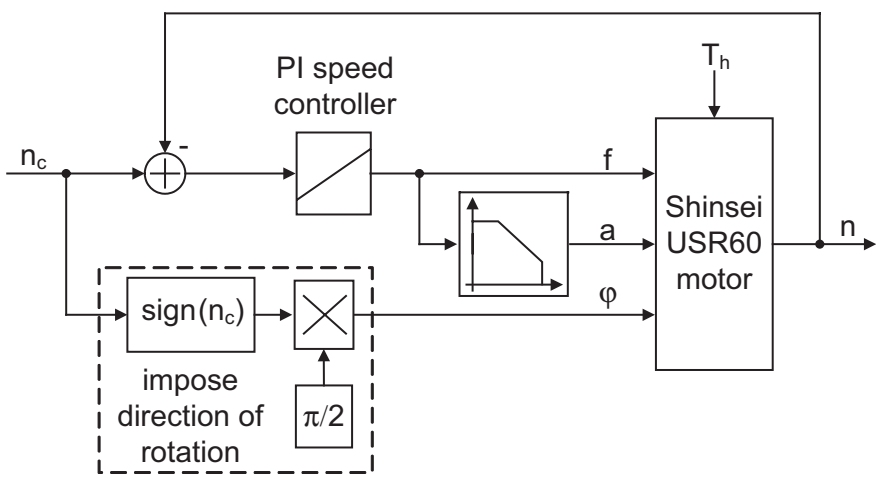

Fig. 7. The speed controller commands frequency $f$, amplitude $a$ and phase shift $\phi$ of the two supply voltages.

The comparison of the speed-frequency characteristics obtained with varying or constant amplitude (figure 8) shows indeed that this control approach results in a linearization in the region of low speed.

The phase shift is fixed at $\frac{\pi}{2}$ to maintain the optimal traveling wave. A change of sign inverts the direction of rotation. These considerations result directly in the control 


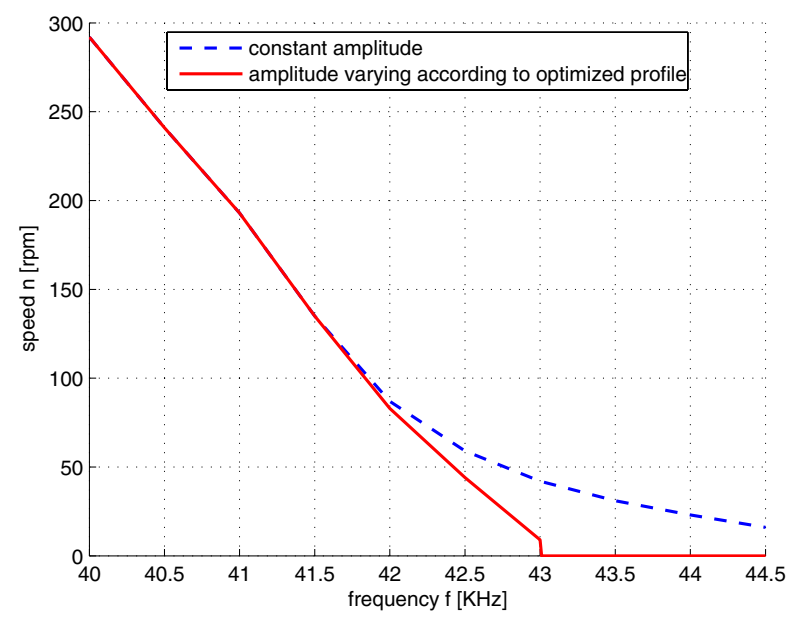

Fig. 8. With constant voltage amplitude, the speed-frequency characteristic is highly non-linear. When the amplitude is optimized, this characteristic becomes linear.

loop shown in figure 7. Experimental validation shows the dynamic behavior of this system to be essentially the same at low and high speed (figure 9).

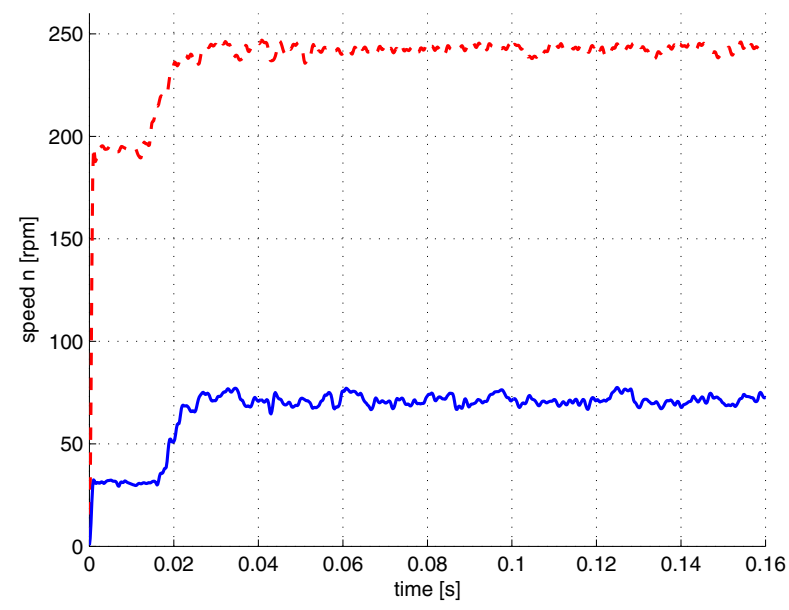

Fig. 9. The dynamic behaviour of the controller is basically the same at low speeds (where the voltage amplitude is varied as a function of frequency) and at high speeds where the voltage amplitude is constant.

Besides the evident advantages of the simplicity of our approach compared to the methods cited above, control by amplitude modulation of the supply voltage allows, in an intrinsic way, to considerably reduce the phase current. The measures reported in figure 10 give evidence of considerably reduced power consumption. For instance, at a speed of $50 \mathrm{rpm}$, current and voltage are lowered by approximately $26 \%$, which corresponds to a reduction in power consumption of about $46 \%$ per phase.

We can thus conclude that the suggested control approach is, among the cited alternative solutions, the one that performs

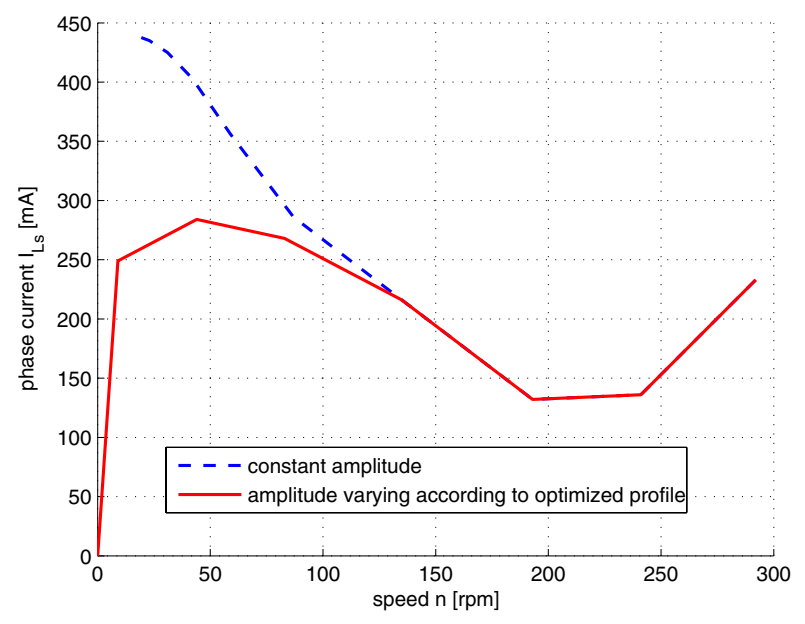

Fig. 10. Evolution of the phase current $I_{L s}$ as a function of motor speed $n$. The phase current is significantly lowered at low speed when the voltage amplitude varies according to the optimized profile.

best in the criteria of performance, robustness, effectiveness and simplicity of implementation.

\section{Position Controller}

To design a position controller we exploit the intrinsic property of the TUSM, which is its holding torque. Contrary to standard servo drives, there will be no power consumption when the commanded position is reached. The controller is thus designed to calculate the approaching speed dependent on the actual positioning error. We notice that when the error of position is higher than a certain threshold, speed is commanded such that the motor is working close to the resonant frequency where efficiency is maximum. When the positioning error falls below the threshold, speed is decreased linearly until the commanded position is reached and feed is cut. In this way we avoid exciting eigenfrequencies of higher order which could damage the mechanics.

\section{Virtual Environment}

The virtual environment we want to represent with the haptic interface is given through its mecanical equivalent in figure 11 .

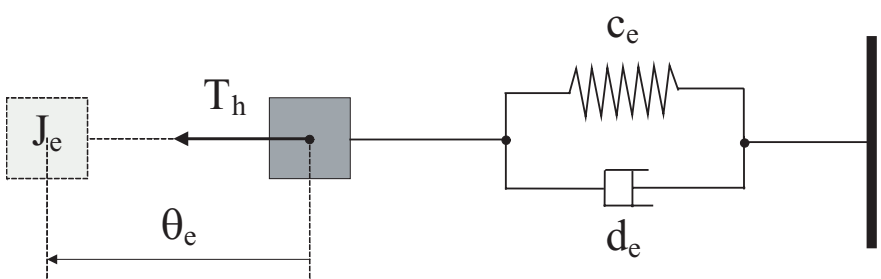

Fig. 11. Equivalent mechanical model of the virtual environment.

Noting its equivalent inertia as $J_{e}$, dumping as $d_{e}$ and stiffness as $c_{e}$, the dynamics of the virtual environment can be represented by equation 2 . 


$$
J_{e} \ddot{\theta}_{e}=T_{h}-d_{e} \dot{\theta}_{e}-c_{e} \theta_{e}
$$

The Laplace transform of equation 2 is given by

$$
\theta_{e}(s)=\underbrace{\frac{1}{J_{e} s^{2}+d_{e} s+c_{e}}}_{Y_{e}} T_{h}(s)
$$

\section{E. Implementation}

The virtual environment is described by a mathematical model implemented on the DSP microprocessor. For validation of the control algorithm we implement the virtual environment given by the second order differential equation 2. For this purpose we need to obtain its discrete form. This is done by approximating the first derivation (angular speed) and second derivation (angular acceleration) as given by equations 4 and 5 .

$$
\begin{gathered}
\dot{\theta}(h)=\frac{\theta(h)-\theta(h-1)}{\Delta h} \\
\ddot{\theta}(h)=\frac{\theta(h)-2 \theta(h-1)+\theta(h-2)}{\Delta h^{2}}
\end{gathered}
$$

With this method, replacing equations 4 and 5 in equation 2 , equation 6 yields the commanded angular position at sample time $h$.

$$
\theta_{e c}(h)=A\left(T_{h}(h)\right)+B\left(\theta_{e}(h-1)\right)+C\left(\theta_{e}(h-2)\right)
$$

Where $\mathrm{A}, \mathrm{B}$ and $\mathrm{C}$ are given in function of the system parameters $J_{e}, c_{e}, d_{e}$ and $\Delta h$ :

$$
\begin{aligned}
& A=\frac{\Delta h^{2}}{J_{e}+\Delta h^{2} c_{e}+\Delta h d_{e}} \\
& B=\frac{\Delta h d_{e}+2 J_{e}}{J_{e}+\Delta h^{2} c_{e}+\Delta h d_{e}} \\
& C=\frac{-J_{e}}{J_{e}+\Delta h^{2} c_{e}+\Delta h d_{e}}
\end{aligned}
$$

As the chosen equivalent mechanical model of the virtual environment is a second order system, it is natural that the commanded angular position $\theta_{e c}(h)$, besides depending on the applied torque, is a function of the measured position at the preceding sample times $\left(\theta_{e c}(h-1)\right.$ and $\left.\theta_{e c}(h-2)\right)$.

The suggested control approach is of a general nature and finds a wide range of applications wherever interaction with human motion is demanded (research, surgery, rehabilitation etc.).

\section{EXPERIMENTAL RESULTS}

The validation of the proposed haptic interface consists on the one hand of a test of the torque feedback control algorithm, including both, conventional performance evaluation as well as the appreciation of a human operator. On the other hand, its compatibility with fMRI must be verified.

\section{A. Validation of controller}

Applying the admittance control strategy, we obtained the results shown in figure 12 . A torque $T_{h}$ is applied artificially by the means of the control electronics to simulate a human operator. The measure of the angular motor position commanded by the control algorithm is then compared to the angular motor position computed on the basis of equation 2 . In addition to the position, the control parameters frequency $(f)$, amplitude $(a)$ and phase shift $(\phi)$ are shown. We observe that the measured position follows the simulated value, which means that the haptic interface reproduces the virtual target environment correctly.

\section{B. EMC test results}

The radiation of the prototype as an isolated system was measured. In a anechoic chamber (electromagnetically shielded room), an annular antenna ${ }^{2}$ was positioned $3 m$ away from the robot as specified in the norms for electromagnetic compatibility measures for medical equipment. The electromagnetic field was measured with a spectrum analyzer ${ }^{3}$. No parasite signal could be observed for the frequency range $(9 \mathrm{kHz}$ to $300 M H z)$ of interest.

\section{MR Compatibility Tests}

Magnetic compatibility is a necessary condition for our system and has been shown with EMC tests. The criteria for MR compatibility are the loss of homogeneity of the magnetic field and the signal to noise ratio (SNR) of the image. Preliminary tests at the University Hospital of $\mathrm{Basel}^{4}$ have shown that no variation in imaging quality due to robot/motor activity can be observed (figure 13). Further, as the same motor/sensor configuration as in [3] is used, it can be said, that the same conclusions concerning the MR combatibility can be made. Similarly, the heating effect has been proven to be minor as shown in previous work [17]. As functional imaging sequences are generally more sensitive to perturbations of the magnetic field than standard diagnostic sequences, we plan to conduct tests with human subjects performing a cognitive task during functional imaging in order to validate the correct behavior of the system, as was suggested in [18].
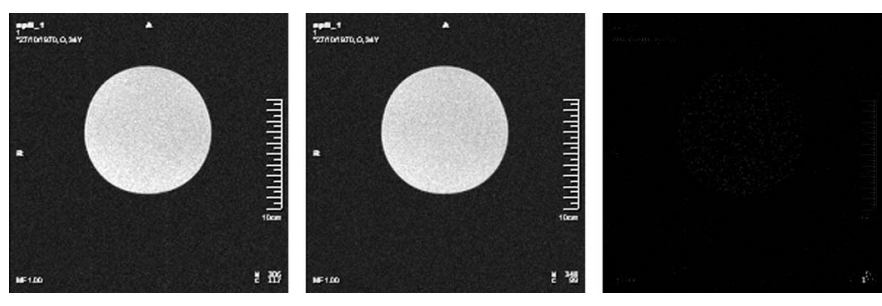

Fig. 13. Functional images of the cylindrical phantom when the robot was not inside the shielded room (left) and when the motor was in motion at the entry of the scanner bore (center). The subtraction of these images shows no shift (right)

\footnotetext{
${ }^{2}$ HLA6120, Schaffner-Chase

${ }^{3} \mathrm{E} 74019 \mathrm{kHz}-1.5 \mathrm{GHz}$ EMC Analyzer, Agilent Technologies

${ }^{4}$ with a Magnetom Espree Tim [76 x 18] MR scanner, Siemens
} 


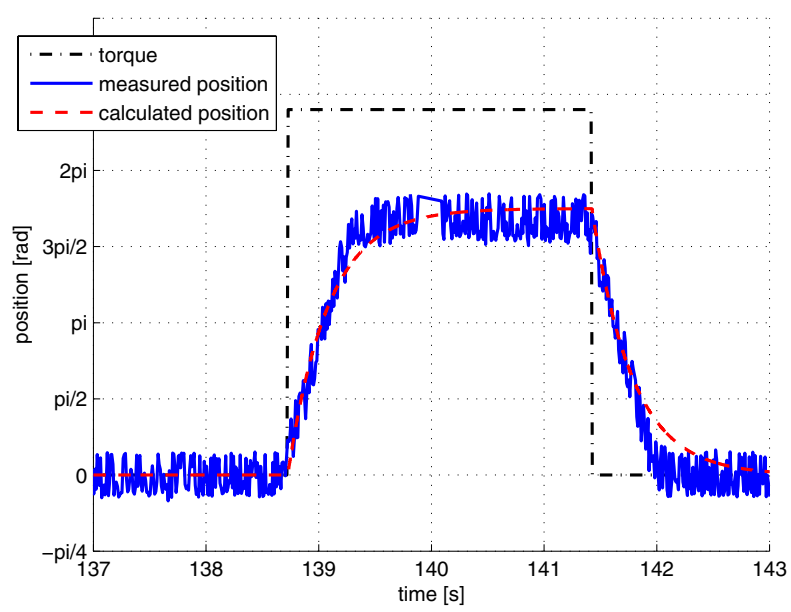

(a) Applied torque, simulated and measured position

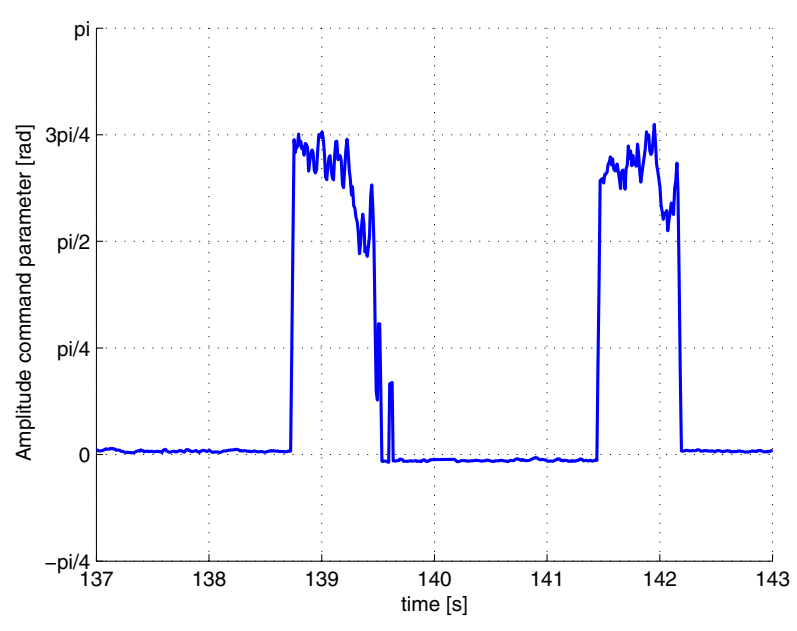

(c) Amplitude command parameter

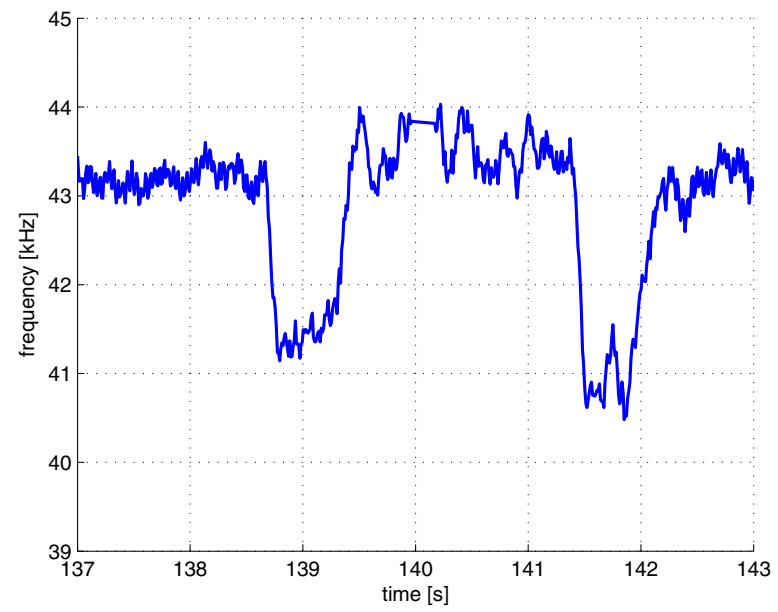

(b) Frequency

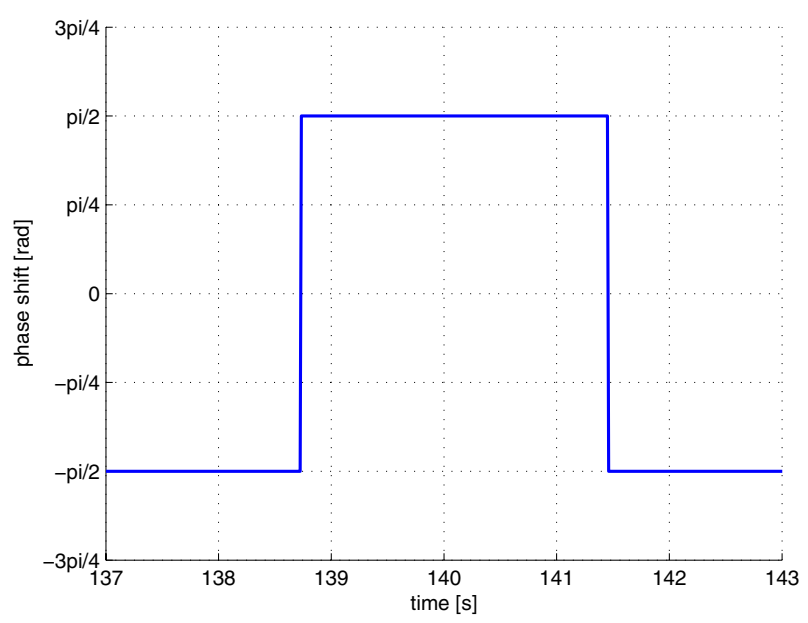

(d) Phase shift between the two supply voltages

Fig. 12. The behavior of the controller was tested with the virtual environment model given in section III-D.

\section{CONCLUSION}

Each component of the system was designed and optimized regarding to fRMI compatibility. The tailormade control algorithm allowed us to optimize the operating characteristics of the TUSM, which initially has a highly nonlinear behavior. The proposed admittance control scheme (section III-A) extends the field of application of TUSM to fMRI compatible haptic interfaces, which gives way to several improvements compared to existing devices that use a hydrostatic transmission concept [18], [5] :

- By the benefit of electrical power transmission, more compact and mechanically simpler solutions can be achieved. Hence, the construction of multiple degree of freedom systems is facilitated.

- Due to the higher bandwidth, the simulation of a larger variety of force fields is possible.
Nevertheless, the multidisciplinarity of this project still offers us many opportunities for future developments. They will be directed towards optimization of the power and control electronics and the realization of a more robust and more compact mechanical structure. Moreover we intend to base further developments of the control method on sensorless technology.

\section{A. Speed Observer}

The position encoder we use does not allow an accurate calculation of low speed and therefore, in the next generation prototype, it will be replaced by a speed estimation algorithm. This allows to reduce cost and dimensions of the haptic interface. Especially, no electronic circuitry will be present in the shielded MR room and compatibility will hence be improved further. A neural network algorithm is chosen for the purpose of speed estimation because of the nonlinearity of the motor characteristics and the fact that no valid analytical model 


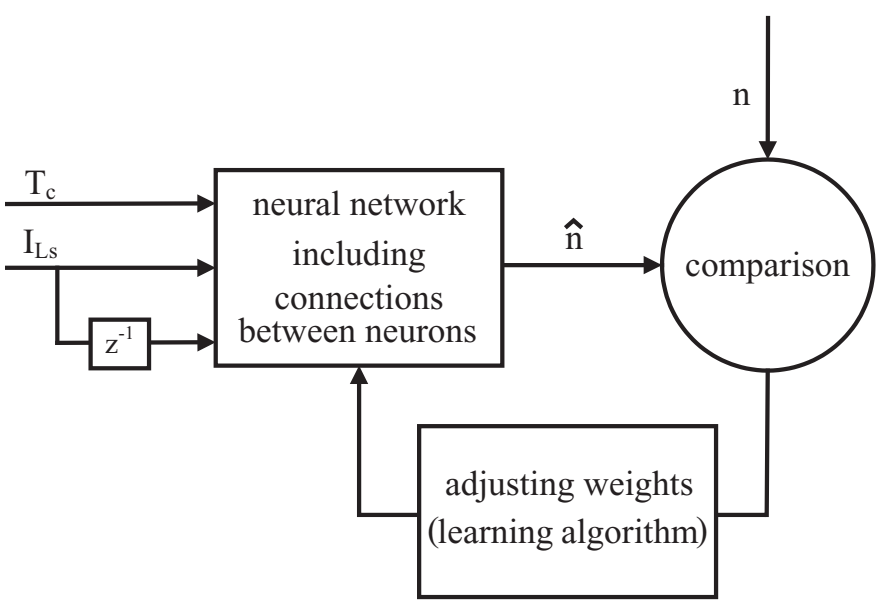

Fig. 14. Schematic representation of the training method for the neural network used to estimate motor speed $\hat{n}$.

can be obtained yet. A neural network, given that enough information is supplied, can be trained to learn any function. Inspired by biologic nervous systems, it is composed of simple elements (neurons) working in parallel which are linked by weighted connections. These weights are adjusted during training in order to minimize the error between measured and estimated output as illustrated in figure 14 .

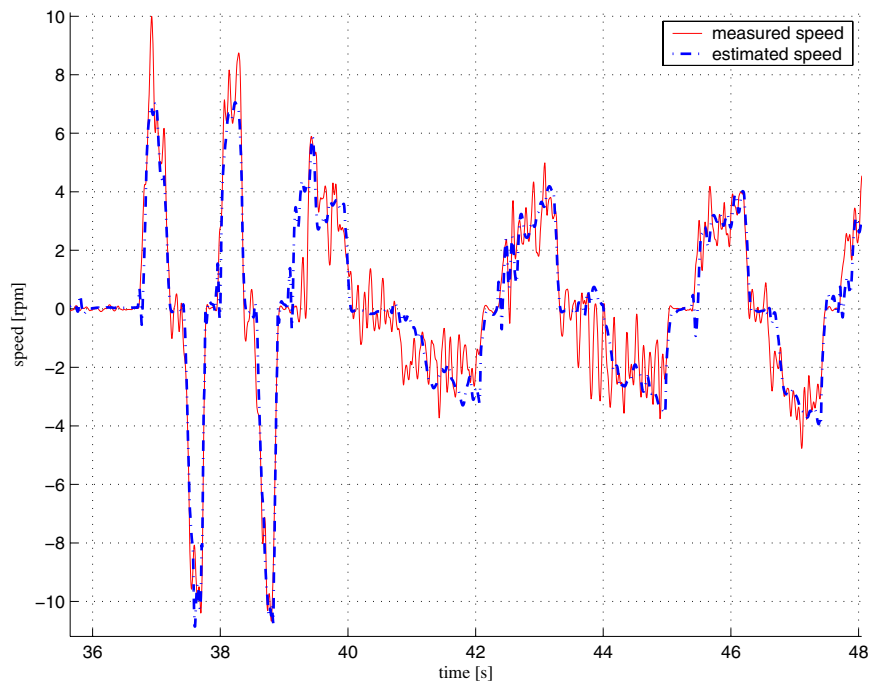

Fig. 15. Results of preliminary speed estimation tests.

Observations during preliminary tests showed that motor speed $n$ is related unequivocally to load torque $T_{c}$ and phase current $I_{L s}$. The torque sensor is necessary because of the TUSM's irreversibility and can therefore also be used as input for the neural network. The neural network will be trained offline on the basis of representative measures aiming to identify this relation. After the training step, the online simulation of the network, excited with measurements of load torque and phase current, will enable us to estimate speed. In order to allow the identification of the system's dynamic behavior, the phase current of the preceding sample step is also an input variable. Position will be obtained by integration. Preliminary results obtained with this method show its feasibility (figure 15). However, the robustness and the stability of the suggested estimation algorithm strongly depends on the information contained in the data used for the training of the neural network. For instance, we noticed a reduced estimation quality when the user of the interface changed. We need to exploit our good knowledge of the TUSM to build a database that allows an effective training of the neural network. A series of tests, which are representative for the typical working conditions, will finally validate robustness and stability of the speed estimation algorithm.

\section{NOMENCLATURE}

$Y_{e} \quad$ Virtual environment admittance

$T_{h} \quad$ Torque applied by human

$T_{m} \quad$ Torque measured by torque sensor

$\theta_{e} \quad$ Virtual environment motor position

$\theta_{e m} \quad$ Measured motor position

$\theta_{e c} \quad$ Commanded motor position

$\dot{\theta}_{e} \quad$ Virtual environment motor speed

$\ddot{\theta}_{e} \quad$ Virtual environment motor acceleration

$J_{e} \quad$ Virtual environment inertia

$d_{e} \quad$ Virtual environment dumping

$c_{e} \quad$ Virtual environment stiffness

$n_{c} \quad$ Commanded motor speed

$n_{m} \quad$ Measured motor speed

$f \quad$ Frequency

a Amplitude command parameter

$\phi \quad$ Phase shift

$I_{L s} \quad$ Phase current

$h \quad$ Sampling time

$\Delta h \quad$ Integration step

$[\mathrm{rad} / \mathrm{Nm}]$
$[\mathrm{Nm}]$
$[\mathrm{Nm}]$
$[\mathrm{rad}]$
$[\mathrm{rad}]$
$[\mathrm{rad}]$
$[\mathrm{rad} / \mathrm{s}]$
$\left[\mathrm{rad} / \mathrm{s}^{2}\right]$
$[\mathrm{N}]$
$[\mathrm{Ns} / \mathrm{m}]$
$[\mathrm{N} / \mathrm{m}]$
$[\mathrm{rpm}]$
$[\mathrm{rpm}]$
$[\mathrm{kHz}]$
$[\mathrm{rad}]$
$[\mathrm{rad}]$
$[\mathrm{A}]$
$[\mathrm{s}]$
$[\mathrm{s}]$

\section{ACKNOWLEDGMENT}

This project was funded by the Swiss National Science Foundation, FNS-205321-104292/1, and the Ecole Polytechnique Fédérale de Lausanne, Switzerland. The authors would like to thank Dr. Markus Klarhöfer and Dr. Sven Haller at the University Hospital of Basel who gave us the opportunity of testing our haptic interface under realistic conditions.

\section{REFERENCES}

[1] D. D. Stark and W. G. Bradley, Magnetic Resonance Imaging. C.V. Moshby Co., St.Louis, 1988.

[2] D. J. Heeger and D. Rees, "What does the fmri tell us about neuronal activity ?" Nature Reviews, no. 3, pp. 142-151, 2002.

[3] K. Chinzei, N. Hata, F. Jolesz, and R. Kikinis, "Surgical assist robot for the active navigation in the intraoperative MRI : Hardware design issues," in Proc. IEEE/RSJ International Conference on Robotics and Intelligent Systems (IROS), 2000, pp. 727-732.

[4] K. Masamune, E. Kobayashi, Y. Masutani, M. Suzuki, T. Dohi, H. Iseki, and K. Takakura, "Development of an MRI-compatible needle insertion manipulator for stereotactic neurosurgery," Journal of Image Guided Surgery, vol. 1, no. 4, pp. 242-248, 1995.

[5] E. Burdet, R. Gassert, G. Gowrishankar, D. Chapuis, and H. Bleuler, "fMRI compatible haptic interfaces to investigate human motor control," Proceedings of the 9th International Symposium on Experimental Robotics (ISER), June 2004 
[6] E. Burdet, R. Osu, D. Franklin, T. Milner, and M. Kawato, "The central nervous system stabilizes unstable dynamics by learning optimal impedance," Nature, no. 414, pp. 446-449, 2001.

[7] R. Shadmehr and H. H. Holcomb, "Neural correlates of motor memory consolidation," Science, vol. 277, no. 5327, pp. 821-825, 1997.

[8] H. I. Krebs, "Increasing productivity and quality of care : Robot aided nuero-rehabilitation," Journal of Rehabilitation Research and Development, vol. 37, no. 6, 2000.

[9] K. Chinzei, R. Kikinis, and A. Jolesz, "MR compatibility of mechatronic devices : Design criteria," Proc. Second International Conference on Medical Image Computing and Computer-assisted Interventions (MICCAI), pp. 1020-31, September 1999.

[10] "System and method for medical imaging utilizing a robotic device, and robotic device for use in medical imaging," Patent US \# 5 794 621, 1995.

[11] B. Larson, A. Erdman, N. Tsekos, E. Yacoug, P. Tsekos, and I. Koutlas, "Design of an MRI-compatible robotic stereotactic device for minimally invasive interventions in the breast," Journal of Biomechanical Engineering, vol. 126, no. 4, pp. 458-65, August 2004.

[12] D. Chapuis, R. Gassert, L. Sache, E. Burdet, and H. Bleuler, "Design of a simple MRI/fMRI compatible force/torque sensor," IEEE International Conference on Robotics and Intelligent Systems (IROS), 2004.

[13] T. Senjyu, K. Uezato, and H. Miyazato, "Adjustable speed control of ultrasonic motors by adaptive control," IEEE Transactions on Power Electronics, vol. 10, no. 5, pp. 532-538, 1995.

[14] F.-J. Lin, "Fuzzy adaptive model-following position control for ultrasonic motor," IEEE Transactions on Power Electronics, vol. 12, no. 2, pp. 261-268, 1997.

[15] T. Senjyu, K. Uezato, S. Yokonda, and H. Miyazato, "Speed control of ultrasonic motors using neural network," IEEE Transactions on Power Electronics, vol. 13, no. 3, pp. 381-387, 1998.

[16] F. J. Lin, W. J. Hwang, and R. J. Wai, "Ultrasonic motot servo-drive with online trained neural-network model-following controller," vol. 145, 1998, pp. 105-110.

[17] R. Buchili, P. Boesiger, and D. Meier, "Heating effects of metallic implants by mri examinations," Magnet Reson Med, no. 7, pp. 255-261, 1988.

[18] R. Moser, R. Gassert, E. Burdet, L. Sache, H. Woodtli, J. Erni, W. Maeder, and H. Bleuler, "An MR compatible robot technology," in IEEE International Conference on Robotics and Automation (ICRA), September 2003, pp. 670-675. 